\title{
Modulation of intestinal microbiota, morphology and mucin composition by dietary insect meal inclusion in free-range chickens
}

Ilaria Biasato', Ilario Ferrocino², Elena Biasibetti ${ }^{1}$, Elena Grego', Sihem Dabbou', Alessandra Sereno', Francesco Gai ${ }^{3}$, Laura Gasco ${ }^{2,3}$, Achille Schiavone ${ }^{1,4^{*}}$, Luca Cocolin² and Maria Teresa Capucchio ${ }^{1}$

\begin{abstract}
Background: Gut health in poultry depends on the balance between the host, intestinal microbiota, intestinal microscopic features and diet. The effects of insect meal (a promising alternative protein source for poultry feed) on chicken gut morphology have recently been reported, but no data about intestinal microbiota and mucin composition modulation are available. The present study evaluated the effects of dietary Tenebrio molitor (TM) meal inclusion on gut health of free-range chickens by intestinal microbiota, morphology and mucin composition characterization.

Results: One hundred forty female medium-growing hybrids were divided into 2 dietary treatments (control feed [C] and 7.5\% TM inclusion, with 5 replicate pens/treatment and 14 birds/pen) and slaughtered at 97 days of age (2 birds/ pen for a total of 10 chickens/diet). The gut microbiota assessment on cecal content samples by 165 rRNA amplicon based sequencing showed higher alpha (Shannon, $P<0.05$ ) and beta (Adonis and ANOSIM, $P<0.001$ ) diversity in birds fed TM diet than C. In comparison with C group, TM birds displayed significant increase and decrease, respectively, of the relative abundances of Firmicutes and Bacteroidetes phyla, with higher Firmicutes:Bacteroidetes ratios (False Discovery Rate $[\mathrm{FDR}]<0.05)$. The relative abundance of Clostridium, Oscillospira, Ruminococcus, Coprococcus and Sutterella genera was higher in TM chickens than C (FDR < 0.05). On the contrary, TM birds displayed significant decrease of the relative abundance of Bacteroides genus compared to the $C$ group (FDR $<0.05$ ). Gut morphology evaluation by morphometric analysis on small intestine revealed similar villus height, crypt depth and villus height to crypt depth ratio between $C$ and TM birds. Characterization of gut mucin composition by periodic-acid Schiff, Alcian Blue pH 2.5 and high iron diamine staining on small and large intestine showed unaffected mucin staining intensity in TM chickens when compared to $\mathrm{C}$ group.
\end{abstract}

Conclusions: Dietary TM meal inclusion may positively modulate the gut microbiota of the free-range chickens without influencing the intestinal morphology and mucin composition. Since the rapid growth of chickens directly depends on morphological and functional integrity of the digestive tract, the gut health assessment by a post mortem multidisciplinary approach appears to be fundamental.

Keywords: $16 \mathrm{~S}$ rRNA, Gut health, Microbiota, Morphometry, Mucin, Poultry, Tenebrio molitor

\footnotetext{
* Correspondence: achille.schiavone@unito.it

'Department of Veterinary Sciences, University of Turin, Largo Paolo Braccini

2, 10095 Grugliasco, TO, Italy

${ }^{4}$ Institute of Multidisciplinary Research on Sustainability, University of Turin,

Via Accademia Albertina 13, 10100 Turin, Italy

Full list of author information is available at the end of the article
}

(c) The Author(s). 2018 Open Access This article is distributed under the terms of the Creative Commons Attribution 4.0 International License (http://creativecommons.org/licenses/by/4.0/), which permits unrestricted use, distribution, and reproduction in any medium, provided you give appropriate credit to the original author(s) and the source, provide a link to the Creative Commons license, and indicate if changes were made. The Creative Commons Public Domain Dedication waiver (http://creativecommons.org/publicdomain/zero/1.0/) applies to the data made available in this article, unless otherwise stated. 


\section{Background}

Gut health has been a focus of major research efforts in production animals, since it can be considered a synonymous to animal health and is of vital importance to animal performance [1]. The latter aspect has a key role in poultry industry, which requires animals capable of growing rapidly within a short period of time.

Gut health depends on the maintenance of the delicate balance between the host, intestinal microbiota, intestinal barrier (in terms of microscopic structure) and dietary compounds [2]. In particular, gut microbiota benefits the host by providing nutrients from otherwise poorly utilized dietary substrates and modulating the development and function of the digestive and immune system [3]. Firstly, gut microbiota can affect intestinal morphology through modifications of villus height and crypt depth [4], which are considered the main indicators of gut development, health and functionality [5]. There is also evidence that gut microbiota may modulate synthesis and composition of mucins [4], which constitute a digestion- and absorption-assisting medium and represent the first line of defense for intestinal epithelium against foreign bacteria and other pathogens [6]. Several feed substances have been reported to widely affect gut health in poultry, either by directly modifying intestinal morphology $[7,8]$ and mucin composition $[9,10]$, or indirectly by modulating intestinal microbiota $[3,11]$.

Feed may be considered the most important entity in the poultry industry in terms of animal health and producers revenue and profit. As a first aspect to consider, feed substances are capable of exposing the birds to potentially harmful organisms and/or components through the gastrointestinal tract [12]. Feed is also the major component of the total cost of production, with protein sources representing the primary one [13]. Corn and soybean meal constitute the main ingredients of choice for poultry diets worldwide [12], but the search for more sustainable, less food-competing and alternative protein feedstuffs is progressively increasing [13].

Insect meal utilization as alternative protein source for animal feeding has already been pointed out [14, 15], because of the excellent nutritive properties, the low competitiveness with human food and the reduction of the environmental contamination [15]. Compared to the conventional protein feedstuff (in particular soybean meal), insects contain higher or similar protein content, being also richer in essential amino acids [14, 15]. Furthermore, mass production of insects is currently promising in an ecological perspective, since it can lead to a significant reduction of the environmental impact in terms of energy cost, land area utilization and footprints. Indeed, insects can easily grow on different organic side streams, whose elimination has an economic and environmental cost. As a consequence, insects rearing may promote an advantageous reutilization of by-products, also transforming waste into high-protein feed that can replace increasingly more expensive compound feed ingredients [15]. Among insect species, meal obtained from larvae of Tenebrio molitor (TM) has recently been tested in diets for broiler [16-20] and free-range chickens [21] as protein source partially or totally replacing the only soybean meal $[16,18]$, soybean meal, corn gluten meal and soybean oil together $[19,20]$, or corn gluten meal alone [21]. In particular, TM larvae meal has been reported to be a suitable dietary ingredient in terms of unaffected or partially improved growth performance, haematochemical parameters, carcass traits and histological features [16, 18-21]. The implications of TM larvae meal utilization have also been investigated on gut health by intestinal morphology assessment [1921], but no data about the modulation of gut microbiota and mucin composition by their inclusion in poultry diets are currently available.

The present study aims to investigate the effects of TM meal utilization on intestinal microbiota, morphology and mucin composition of free-range chickens, also proposing a standardized multidisciplinary post mortem approach for the assessment of gut health in poultry when dietary modifications occur.

\section{Results}

\section{Cecal microbiota characterization}

A total of 530,550 raw reads (2x250bp) were obtained after sequencing. After joint and quality filtering, a total of 104,081 reads passed the filters applied through QIIME, with an average value of 10,408 reads/sample. In order to avoid biases due to different sequencing depths, all samples were rarefied at 3600 reads after raw read quality filtering.

The rarefaction analysis and the Good's coverage indicated a satisfactory coverage for all the samples (average Good's coverage of 84\%) (Additional file 1). The diversity of the cecal microbiota between $C$ and TM diets was assessed initially through measures of $\alpha$-diversity and $\beta$-diversity. The Chao1, Phylogenetic Diversity (PD) Whole Tree and Shannon indexes and observed species richness were used to assess $\alpha$-diversity (Additional file 1 ). Shannon index showed higher diversity $(P<0.05)$ in free-range chickens fed with TM compared to $\mathrm{C}$ diet, whereas Chao1 and PD Whole Tree indexes and observed species richness showed no significant differences $(P>0.05)$ between $C$ and TM groups (Additional file 1). Weighted UniFrac distances were utilized as a measure of $\beta$-diversity to assess the effect of TM diet on bacterial community composition. Adonis and ANOSIM statistical tests based on Weighted UniFrac distance matrix showed significant 
differences among $\mathrm{C}$ and TM groups $(P<0.001)$. These differences were demonstrated by Principal Component Analysis (PCA), which showed a clear separation of the microbiota as a function of the diet (Fig. 1).

Relative abundances of the main phyla and genera in the free-range chickens of the present study obtained by $16 \mathrm{~S}$ rRNA gene sequencing are summarized in Fig. 2 (phyla and genera per pools) and Additional file 2 (overall phyla and genera). Bacteroidetes represented the dominant phylum of the cecal community in both $\mathrm{C}$ and TM groups, outnumbering the Firmicutes and Proteobacteria phyla (Fig. 2a, Additional file 2). Within phylum Bacteroidetes, Bacteroides, unclassified members (U. m.) of Bacteroidales order, Alistipes, Parabacteroides and Coprobacter were identified as predominant OTUs in both birds fed with $\mathrm{C}$ and TM diets (Fig. 2b, Additional file 2). Clostridium, Ruminococcus, Oscillospira, L-Ruminococcus, Faecalibacterium and $\mathrm{U}$. m. of Lachnospiraceae family were the dominant members of the Firmicutes phylum in both $\mathrm{C}$ and TM groups (Fig. 2b, Additional file 2). Within phylum Proteobacteria, U. m. of Alphaproteobacteria class and U. m. of Succinivibrionaceae family were observed as predominant OTUs in both chickens fed with $\mathrm{C}$ and TM diets (Fig. 2b, Additional file 2).

Compared to the $\mathrm{C}$ group (Fig. 3), the birds fed with TM displayed significant increase of the relative abundance of Firmicutes phylum $(\mathrm{FDR}<0.05)$. On the contrary, the relative abundance of Bacteroidetes was lower in TM chickens than C (FDR < 0.05). The birds fed with TM also showed higher Firmicutes:Bacteroidetes ratios compared to the $C$ group (FDR < 0.05). At genus level (Fig. 4),

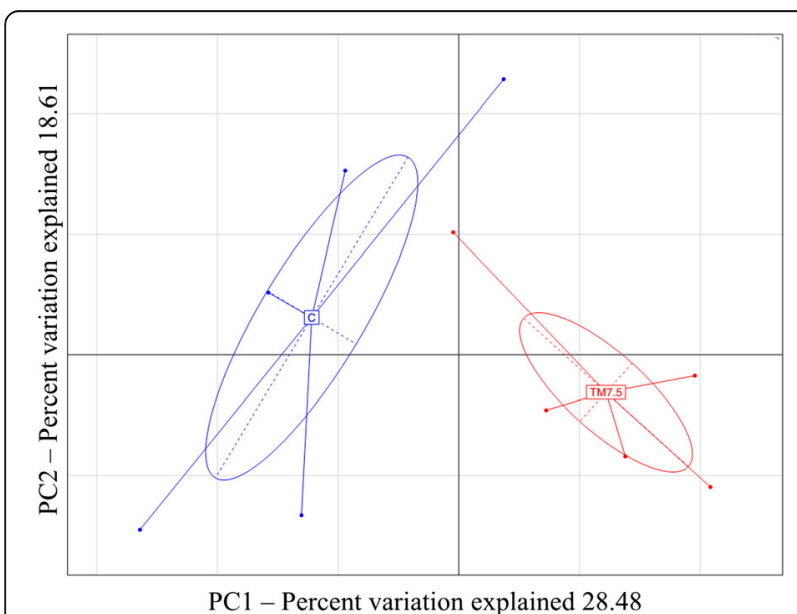

Fig. 1 Bacterial community composition (weighted UniFrac beta diversity, PCA plots) in cecal samples of free-range chickens fed with control (C) and $7.5 \%$ inclusion level of Tenebrio molitor meal (TM7.5) diets. PC1 and PC2 components account for 28.48 and $18.61 \%$, respectively, of the total variation (47.09) the relative abundance of Bacteroides was lower in TM chickens than $C(F D R<0.05)$. On the contrary, the birds fed with TM displayed significant increase of the relative abundance of Sutterella, Ruminococcus, Oscillospira , Clostridium and Coprococcus genera compared to the $\mathrm{C}$ group $(\mathrm{FDR}<0.05)$.

\section{Intestinal morphology}

Dietary TM meal inclusion did not affect the intestinal morphometric indices of the free-range chickens of the present study $(P>0.05$, Fig. 5). Independently of TM meal utilization, the duodenum showed greater Vh $(\mathrm{C}$ and TM7.5 groups, $P<0.01)$ and $C d$ (TM7.5 group, $P<0.05)$ than the other gut segments and higher $\mathrm{Vh} / \mathrm{Cd}$ ratio (C group, $P=0.01$ ) than the ileum. The detailed results of the intestinal morphology of the chickens of the current trial are reported by Biasato et al. [21].

\section{Intestinal mucin composition}

Mucin staining intensity in the intestinal crypts of free-range chickens of the present study significantly depended on mucin type, gut segment and crypt fragment $(P<0.001)$. However, there was no significant influence of dietary TM meal inclusion $(P>0.05)$ on the histochemical findings (Table 1). In particular, crypts showed higher acidic sialylated mucins staining intensity $(P<0.001)$ than neutral and acidic sulfated. Furthermore, lower mucin staining intensity was found in the caecal crypts $(P<0.001)$ compared with the other gut segments and in the jejunal crypts $(P<0.001)$ compared with the duodenum and ileum, respectively. Crypt base also showed greater mucin staining intensity $(P<0.001)$ than the midsection and tip (Table 2, Fig. 6).

There was no significant effect of dietary TM meal inclusion or mucin type $(P>0.05)$ on mucin staining intensity for the intestinal villi, whereas both gut segment and villus fragment significantly influenced $(P<0.001$ and $P<0.01$, respectively) the histochemical findings (Table 1). In particular, villi showed higher mucin staining intensity in the ileum $(P<0.001)$ compared with the other gut segments and in the jejunum $(P<0.001)$ compared with the duodenum, respectively (Fig. 7). Lower mucin staining intensity was also observed in the villus tip $(P=0.001)$ than the base (Table 2$)$.

\section{Discussion}

A clear definition for "gut health" that encompasses all the intestinal physiological and functional features, such as nutrient digestion and absorption, host metabolism and energy generation, a stable microbiome, mucus layer development, barrier function, and mucosal immune responses, has never been reported [1]. However, the crosstalk between the diet composition, 


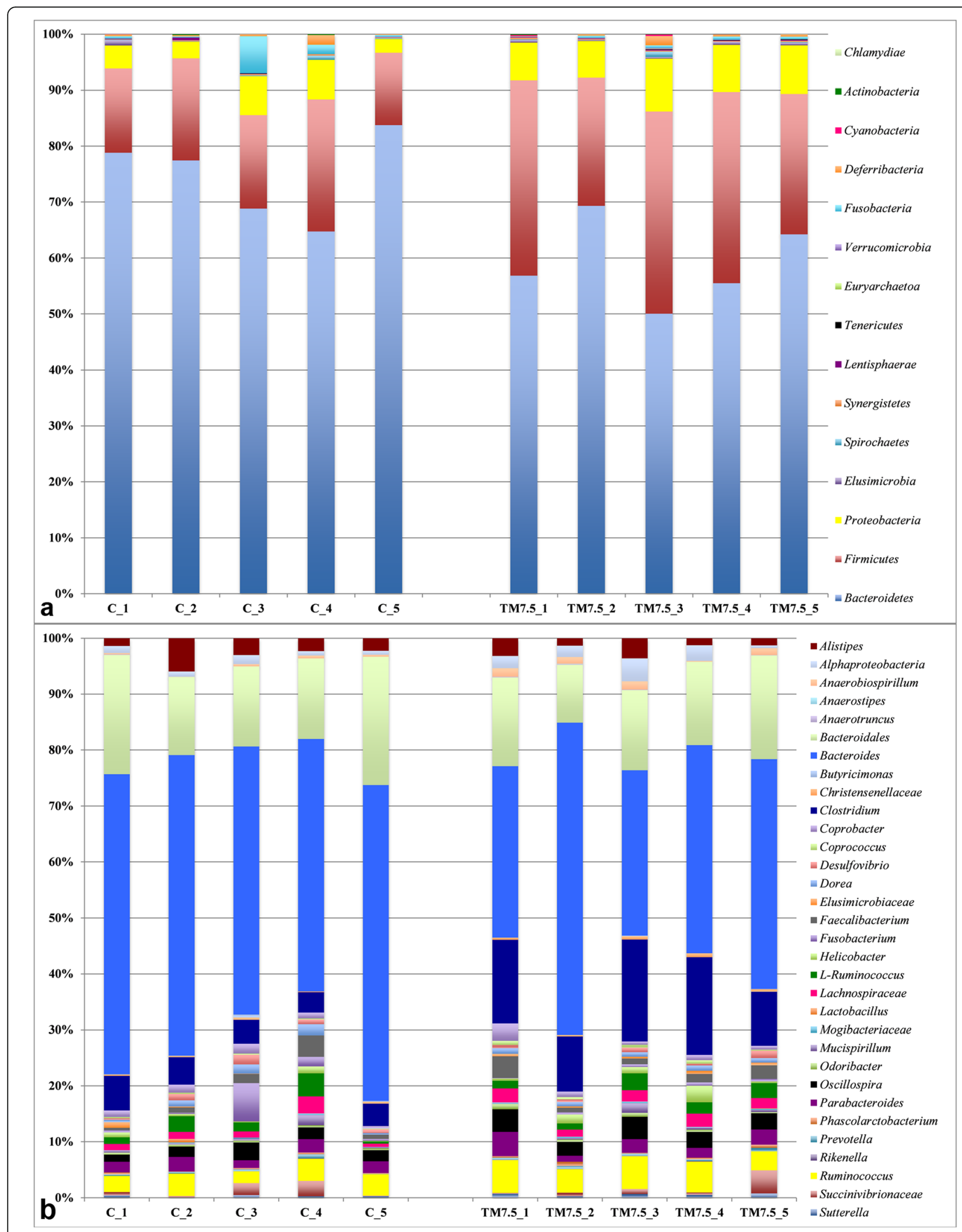

Fig. 2 (See legend on next page.) 
(See figure on previous page.)

Fig. 2 Relative abundance of the main bacterial phyla (a) and genera (b) in cecal samples of free-range chickens fed with control (C) and 7.5\% inclusion level of Tenebrio molitor meal (TM7.5) diets. Taxa summary bar plots within the pooled cecal contents from the 5 replicate pens of control (C_1, C_2, C_3, C_4 and C_5) and 7.5\% inclusion level of Tenebrio molitor meal (TM7.5_1, TM7.5_2, TM7.5_3, TM7.5_4 and TM7.5_5) dietary treatments

intestinal barrier (formed by the mucus layer together with the epithelial cell layer) and intestinal microbiota seems to be crucial for gut health determination [2]. For such reasons, several authors have recently focused their attention on the relationship between gut microbiota and mucin composition, gut microbiota and morphology, or gut morphology and mucin composition when dietary modifications occurred in poultry. However, systematic studies about the assessment of gut health by the evaluation of all the three aspects together (gut microbiota, morphology and mucin composition) in chickens facing to new diets are still very limited. Furthermore, no information about the modulation of intestinal microbiota and mucin composition by dietary insect meal inclusion in poultry are currently available.

\section{Cecal microbiota characterization}

Characterization of the cecal microbiota of free-range poultry has already been reported in previous studies about Chinese Dagu chickens [22], Italian local breeds [23] and Bermuda free-range chickens [24]. However, the present study is the first one investigating the cecal microbiota of Label Hubbard chickens fed with different diets. The attention was herein focused on cecum as representative gut segment, since it harbors the highest microbial cell densities (up to $10^{11}$ cells/g) and diversity, has the longest residence time $(12-20 \mathrm{~h})$ of digesta in the gastrointestinal tract, and is an important site for recycling of urea, water regulation, and carbohydrate fermentations contributing to intestinal health and nutrition [25].

In the free-range chickens fed with basal diet of the present study, cecal microbiota was mainly colonized by

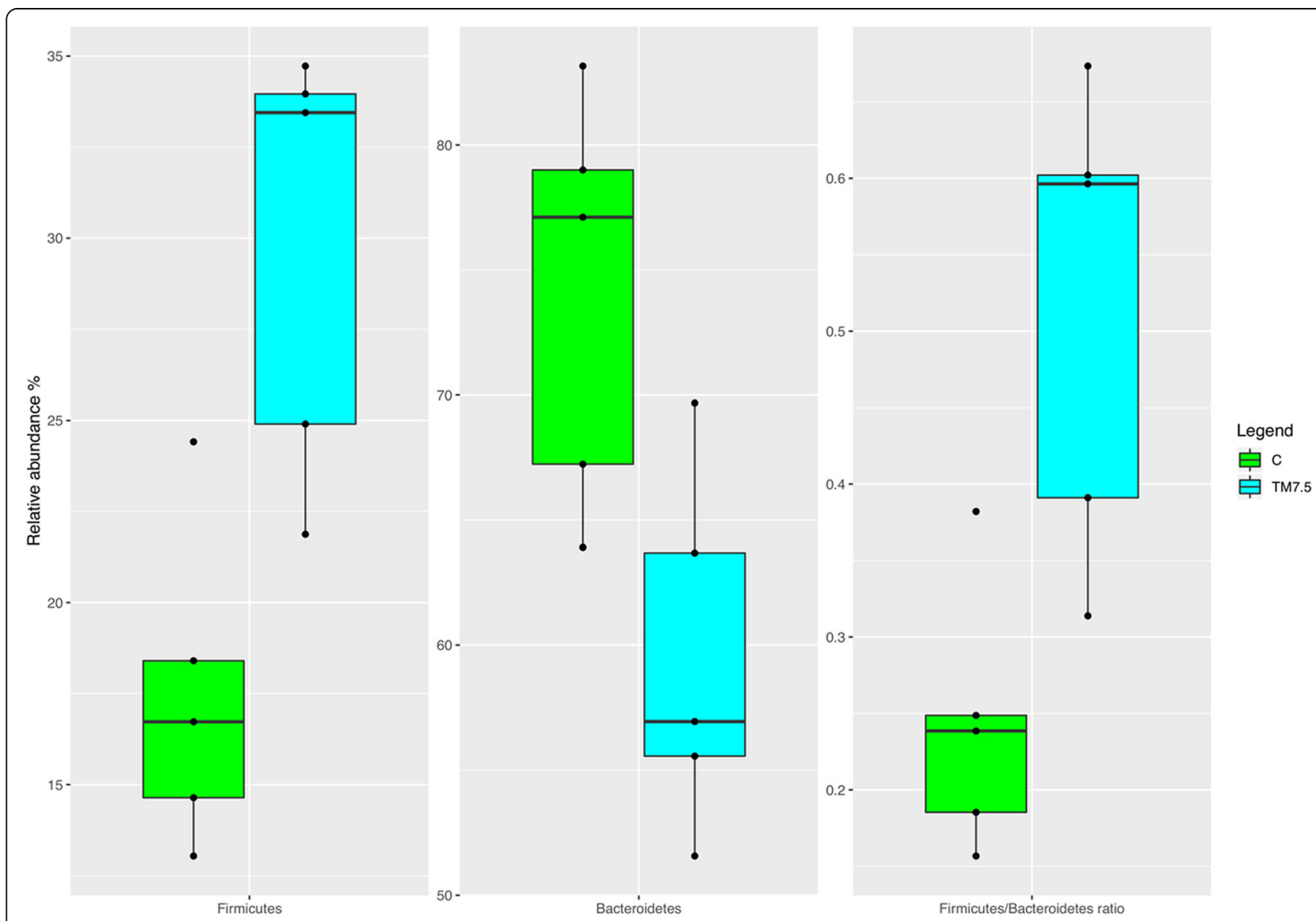

Fig. 3 Boxplots showing the relative abundance at phylum level of OTUs differentially abundant based on Pairwise Kruskal-Wallis test (FDR < 0.05) in cecal samples of free-range chickens fed with control (C) and 7.5\% inclusion level of Tenebrio molitor meal (TM7.5) diets 


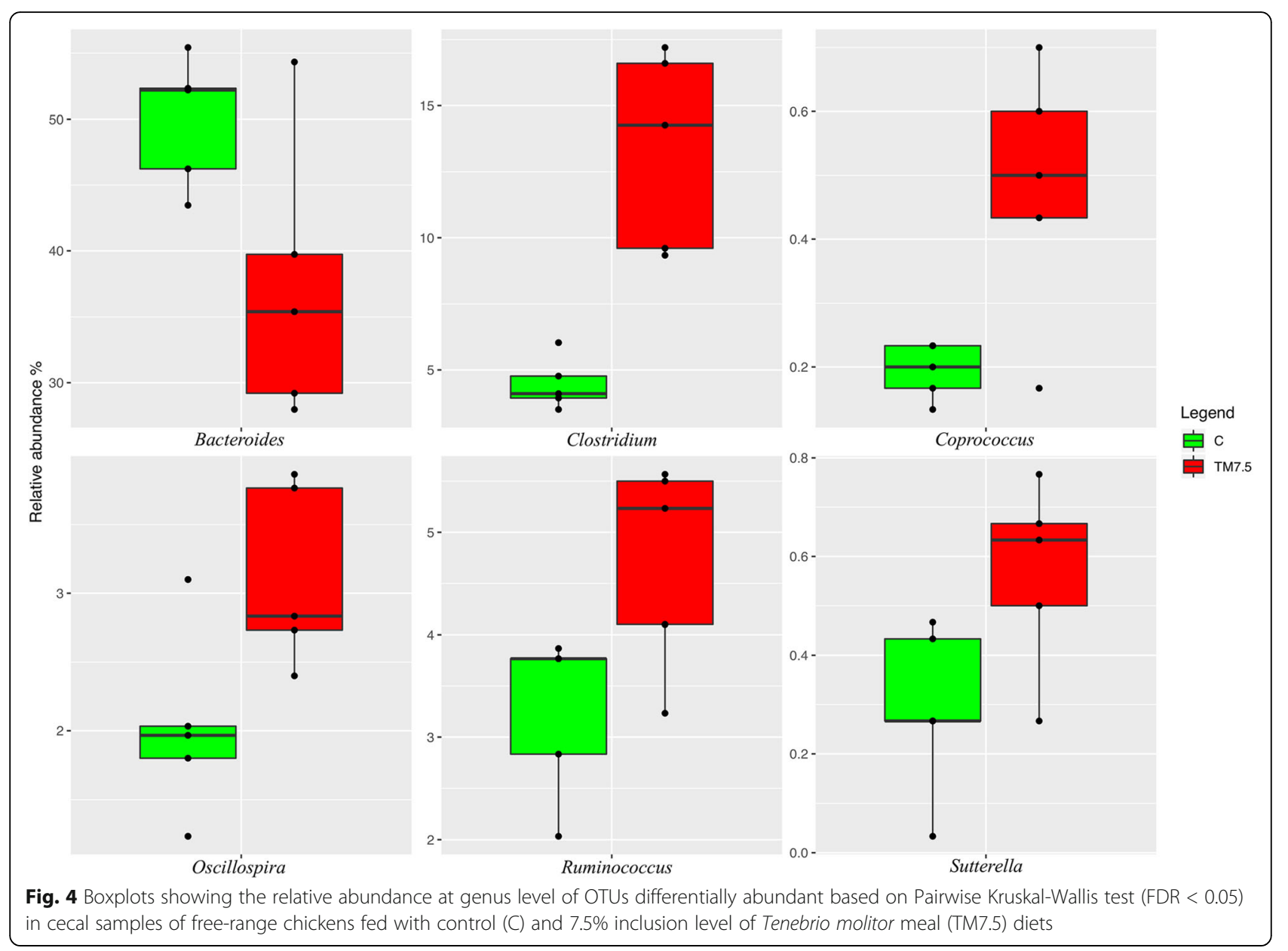

the phyla Bacteroidetes, Firmicutes and Proteobacteria, as previously reported in normal chickens [25-28]. In particular, the predominance of the phylum Bacteroidetes over Firmicutes and Proteobacteria is in agreement with the previous findings about cecal microbiota of Dagu [22] and Bermuda [24] free-range chickens. A more similar prevalence of Bacteroidetes, Firmicutes and Proteobacteria phyla was observed in Italian local free-range breeds, but a comparative reduction of Firmicutes and a concomitant increase of Bacteroidetes was still identified in these birds when compared to broilers [23].

Differently from phyla, at genus level there is some controversy over the predominant taxa in chicken cecal microbiota. The majority of studies observed that the most predominant genera found in chicken cecum are Clostridium, Ruminococcus, Lactobacillus and Bacteroides [28-32], while Callaway et al. [33] also identified Prevotella as the most abundant genus. In particular, Bacteroides genus, U.m. of Prevotellaceae family, U. $\mathrm{m}$. of Ruminococcaceae family and U. m. of Bacteroidales order have been reported as the most predominant members of cecal microbiota of Bermuda free-range chickens [24]. The current trial, which observed Bacteroides, U. m. of Bacteroidales order, Clostridium and Ruminococcus as the most predominant genera, can fit into the overall view of these previous studies.

With regards to gut microbiota modulation by insect meal utilization, the cecal microbiota of the free-range chickens of the present study showed higher $\alpha$ - and $\beta$-diversity when fed with TM meal compared to $C$ feed. This represents a quite relevant finding, since high levels of diversity have been reported to help maintain the stability of intestinal microbiota after environmental stress [34] and determine the colonization resistance against invading pathogens [35]. Another interesting aspect to consider is that the average Shannon index identified in the current trial was about 8 . This is in contrast with the previous studies about the chicken cecal microbiota, which reported a Shannon index varying from 3 to 6 $[36,37]$. Mancabelli et al. [23] already pointed out that free-range chickens displayed a higher level of complexity of the gut microbiota compared to that found in broilers, with a Shannon index slightly higher than 6 . Interestingly, Ferrario et al. [24] also observed similar microbial diversity between free-range and feral (i.e., 


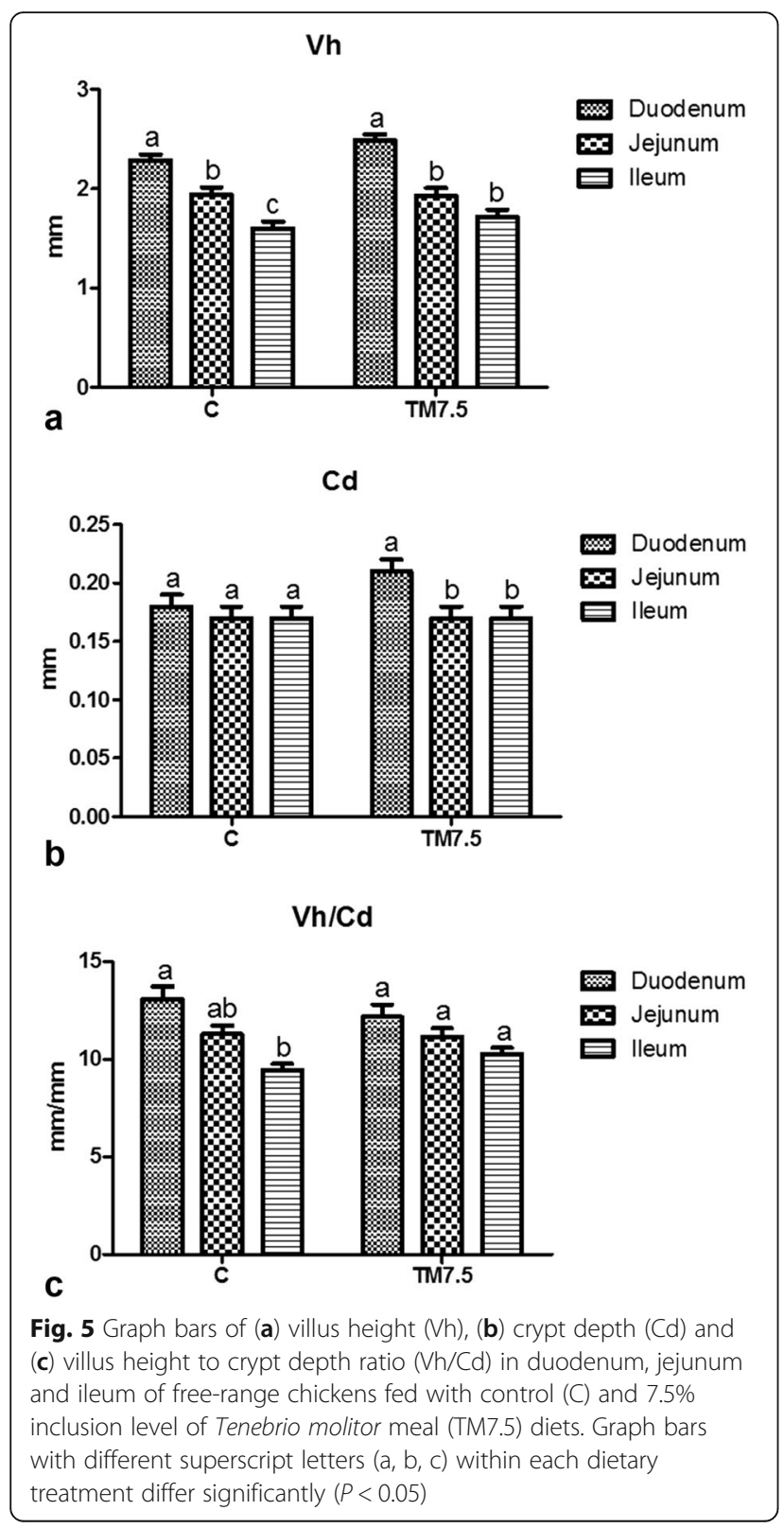

formerly domesticated, wild-living) chickens, with the last being characterized by a Shannon index around 7 . Therefore, it could be hypothesized that the semi-wild and wild rearing conditions may progressively modulate the complexity of the intestinal microbiota, thus underlying the role of environment and human influence on the bacterial communities within the chicken gastrointestinal tract.

Similarly to what observed for $\mathrm{C}$ birds, the cecal microbiota of the free-range chickens fed with TM diet of the present study was mainly colonized by the phyla Bacteroidetes, Firmicutes and Proteobacteria. Furthermore, the free-range chickens fed with TM showed Bacteroides, U. m. of Bacteroidales order, Clostridium
Table 1 Effects of diet, mucin type, gut segment and crypt-villus fragment on mucin staining intensity in free-range chickens

\begin{tabular}{llll}
\hline Factor & d.f. $^{f}$ & Chi-square & P-value $^{g}$ \\
\hline Crypts & & & \\
Diet $^{\mathrm{a}}$ & 1 & 0.77 & $\mathrm{~ns}$ \\
Mucin type $^{\mathrm{b}}$ & 2 & 34.61 & ${ }^{* * *}$ \\
Gut segment $^{\mathrm{c}}$ & 3 & 145.01 & ${ }^{* * *}$ \\
Fragment $^{\mathrm{d}}$ & 2 & 71.38 & \\
Villi & & & \\
Diet & 1 & 0.00 & $\mathrm{~ns}$ \\
Mucin type & 2 & 3.03 & $\mathrm{~ns}$ \\
Gut segment & 2 & 272.02 & ${ }^{* * *}$ \\
Fragment & 2 & 10.99 & ${ }^{* *}$ \\
\hline
\end{tabular}

a Two dietary treatments: $C$ control, TM7.5 7.5\% inclusion level of Tenebrio molitor

b Three types: neutral, acidic sialylated and acidic sulfated mucins

c Four segments: duodenum, jejunum, ileum and cecum

${ }^{d}$ Three fragments: base, midsection and tip

e Three segments: duodenum, jejunum and ileum

${ }^{f}$ Degrees of freedom

${ }^{g}$ Statistical significance: $P<0.05\left({ }^{*}\right), P<0.01\left({ }^{* *}\right)$ and $P<0.001\left({ }^{* * *}\right)$. ns $=$ not significant

and Ruminococcus as the most predominant genera of their cecal microbiota. These findings suggest that TM meal utilization does not alter the physiological cecal community at both phylum and genus levels.

Investigating the compositional differences in the cecal microbiota between $\mathrm{C}$ and TM birds, the free-range chickens fed with insect meal showed increased and decreased abundances of Firmicutes and Bacteroidetes phyla, respectively, along with higher Firmicutes:Bacteroidetes ratios. This represents a quite relevant finding, since bacteria within Firmicutes phylum have an important role in the digestion of feed and the host health [38] and greater Firmicutes:Bacteroidetes ratios have been associated with bacterial profile with higher capacity of energy harvesting [39]. At genus level, the free-range chickens fed with TM showed an increase in the abundance of Clostridium, Oscillospira, Ruminococcus, Coprococcus and Sutterella genera in their cecal community. As already pointed out, Clostridium and Ruminococcus are some of the most predominant genera found in chicken cecum [28-30]. On the other hand, Clostridium genus, along with Oscillospira and Coprococcus, also encompasses bacteria capable of producing butyrate [40, 41]. Butyrate has been demonstrated to have a positive role on growth performance, intestinal villus structure and pathogen control, as well as anti-inflammatory properties [42]. Furthermore, bacteria belonging to Ruminococcus genus can also produce other short chain fatty acids (i.e., acetic and succinic acid) through glucose metabolism and digest cellulose in food [43]. It is well known that short chain fatty acids are an important source of energy for enterocytes and are vital for intestinal health [44]. Another interesting finding of the cecal community of the birds fed 
Table $\mathbf{2}$ Least square means of mucin staining intensity in the intestinal crypts and villi of free-range chickens in relation to diet, mucin type, gut segment and fragment

\begin{tabular}{|c|c|c|c|}
\hline & Factor & Factor levels & $\begin{array}{l}\text { Mucin staining } \\
\text { intensity }{ }^{a, b}\end{array}$ \\
\hline \multirow[t]{12}{*}{ Crypts } & \multirow[t]{2}{*}{ Diet } & C & $1.11 \pm 0.03$ \\
\hline & & TM7.5 & $1.14 \pm 0.03$ \\
\hline & \multirow[t]{3}{*}{ Mucin type } & Neutral & $1.04 \pm 0.03^{b}$ \\
\hline & & Acidic sialylated & $1.28 \pm 0.04^{a}$ \\
\hline & & Acidic sulfated & $1.06 \pm 0.03^{b}$ \\
\hline & \multirow[t]{4}{*}{ Gut segment } & Duodenum & $1.38 \pm 0.04^{\mathrm{a}}$ \\
\hline & & Jejunum & $1.05 \pm 0.04^{b}$ \\
\hline & & Ileum & $1.31 \pm 0.04^{a}$ \\
\hline & & Cecum & $0.83 \pm 0.03^{c}$ \\
\hline & \multirow[t]{3}{*}{ Fragment } & Base & $1.36 \pm 0.04^{\mathrm{a}}$ \\
\hline & & Midsection & $1.03 \pm 0.03^{b}$ \\
\hline & & Tip & $1.01 \pm 0.03^{b}$ \\
\hline \multirow[t]{11}{*}{ Villi } & \multirow[t]{2}{*}{ Diet } & C & $1.65 \pm 0.04$ \\
\hline & & TM7.5 & $1.65 \pm 0.04$ \\
\hline & \multirow[t]{3}{*}{ Mucin type } & Neutral & $1.63 \pm 0.05$ \\
\hline & & Acidic sialylated & $1.72 \pm 0.05$ \\
\hline & & Acidic sulfated & $1.61 \pm 0.05$ \\
\hline & \multirow[t]{3}{*}{ Gut segment } & Duodenum & $1.09 \pm 0.04^{c}$ \\
\hline & & Jejunum & $1.91 \pm 0.06^{\mathrm{b}}$ \\
\hline & & Ileum & $2.16 \pm 0.06^{a}$ \\
\hline & \multirow[t]{3}{*}{ Fragment } & Base & $1.78 \pm 0.05^{\mathrm{a}}$ \\
\hline & & Midsection & $1.64 \pm 0.05^{\mathrm{ab}}$ \\
\hline & & Tip & $1.55 \pm 0.05^{b}$ \\
\hline
\end{tabular}

C control, TM7.5 7.5\% inclusion level of Tenebrio molitor

${ }^{a}$ Data are represented as mean of counts \pm SEM

${ }^{\mathrm{b}}$ Means with different superscript letters $(\mathrm{a}, \mathrm{b}, \mathrm{c})$ within the same column per factor (i.e. diet, mucin type, gut segment or fragment) differ significantly $(P<0.01)$ with TM in the current trial is the identification of increased abundance of Sutterella genus, which has been reported to be a potential probiotic present in pigeon "milk" that can improve the rate of growth and feed conversion ratio in chickens [45]. The increase of the above mentioned bacterial taxa suggests that TM meal utilization may positively modulate the cecal microbiota of birds. In particular, the increase of butyrate- and short chain fatty acids-producing bacteria may have important implications. However, further studies also evaluating the microbial metabolites and metabolic pathways are needed to better contextualize these OTUs changes.

Despite this overall positive modulation, the reduction of Bacteroides genus within the cecal community of the free-range chickens fed with TM meal of the present study may be considered a potential negative finding. Indeed, apart from being one of the most predominant genera found in chicken cecum [28, 31,33], this taxon has been reported to be an important contributor to the intestinal health of the birds, because of its beneficial role for weight gain and growth performance [46] and the inhibition of Clostridium perfringens sporulation by its fermentation products [47]. As a consequence, depletion of Bacteroides genus could be problematic for chickens, since it may predispose the animal gut to Clostridium perfringens infection and gastroenteritis [47]. However, Bacteroides genus still outnumbered the other taxa in TM group of the present study. The birds fed with insects also showed similar growth performance to those fed with $C$ diet [21], thus further mitigating this potential negative result. As a final aspect to underline, in spite of the overall positive cecal microbiota modulation due to TM meal utilization, the growth performance of the chickens remained unaffected. However, since a clear cause-effect relationship between diversity and composition of cecal microbiota and bird performance has not yet been confirmed, the gut microbiota findings need to be contextualized with those related to mucin composition and morphology.

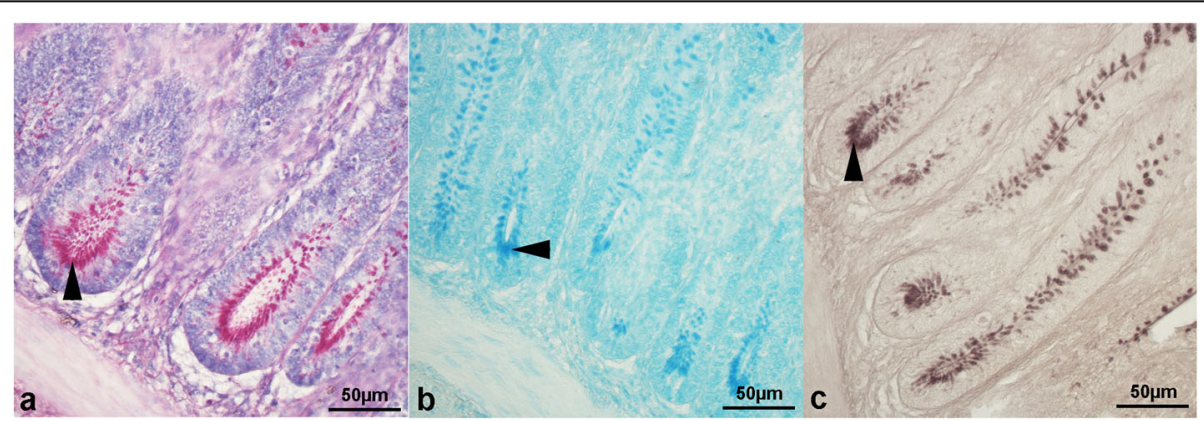

Fig. 6 Histological pictures of (a) duodenal crypts stained with periodic-acid Schiff (40x magnification), (b) jejunal crypts stained with Alcian Blue pH 2.5 (40x magnification) and (c) ileal crypts stained with high iron diamine (40x magnification). Crypt bases (arrowheads) show higher mucin staining intensity than midsection and tip fragments 


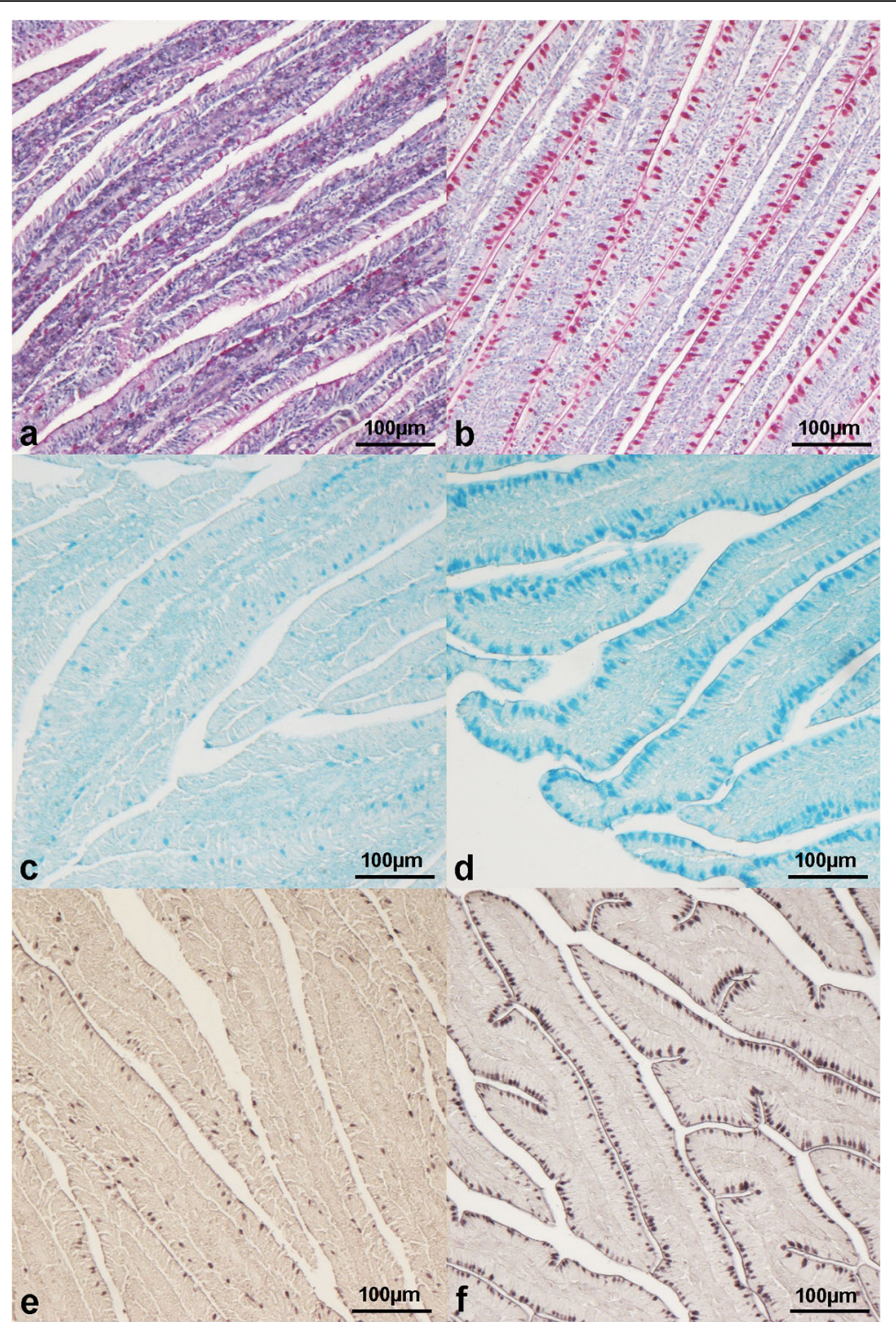

Fig. 7 Histological pictures of duodenal $(\mathbf{a}, \mathbf{c}, \mathbf{e})$ and ileal $(\mathbf{b}, \mathbf{d}, \mathbf{f})$ villi stained with $(\mathbf{a}, \mathbf{b})$ periodic-acid Schiff (10x magnification), (c, d) Alcian Blue $\mathrm{pH} 2.5$ (10x magnification) and (e, $\mathbf{f}$ ) high iron diamine (10× magnification). lleal villi show higher mucin staining intensity than duodenal ones

\section{Intestinal morphology}

Dietary TM meal inclusion in free-range chickens of the current trial did not affect the gut morphometric indices, as already reported in details by Biasato et al. [21]. Features of the gastrointestinal tract have been reported to influence the efficiency of utilization of dietary protein [48], which is considered a crucial regulator of poultry growth and reproductive performance [7]. In particular, the microscopic structure of small intestine in terms of villus height and crypt depth is considered the main indicator of intestinal development, health and functionality, such influencing nutrient digestion and absorption [5]. Particular attention is given to crypt and villus since the former is the region in which new intestinal cells are formed and the latter has a fundamental role in the digestion and absorption of nutrients [48]. The ideal gut morphological asset appears to be characterized by long villi and shallow crypts. Indeed, longer villi are generally associated with increased total luminal absorptive area and subsequent satisfactory digestive enzyme action and higher transport of nutrients [7]. In parallel, shallower crypts reflect the prolonged survival of villi without the need for renewal [49], with reduced energy expenditure for this process and consequent enabled growth of other tissues [50]. Since no standardized measurements ranges referring to "long villi" and "shallow crypts" have been determined till now, the identification of unaffected gut morphometric indices and growth performance in the 
birds fed with insects of the present study is enough to suggest that TM meal does not alter the physiological intestinal morphology and subsequently the digestion efficiency.

Independently of TM meal utilization, the duodenum of the free-range chickens of the current trial showed greater development in relation to the other gut segments. It is well known that the intestine possesses an inherent ability to create and maintain regional differences with regard to mucosal structure [51], thus determining different absorption processes depending on the segment considered [52]. The identification of a proximodistal decreasing gradient of the morphometric indices from the duodenum to the ileum, which is in agreement with the previous studies $[4,52,53]$, is another important aspect in terms of preservation of the physiological gut development and absorption processes.

\section{Intestinal mucin composition}

The present study is the first one to investigate the effects of dietary TM meal inclusion on gut mucin composition in poultry. Furthermore, the characterization of the three types of mucins (i.e., neutral, acidic sialylated and acidic sulfated) in the two intestinal mucosal compartments (i.e., crypts and villi) of four defined segments (i.e., duodenum, jejunum, ileum and cecum) represents a unique histochemical approach.

Dietary TM meal inclusion in free-range chickens of the current trial did not influence the mucin staining intensity either in the intestinal crypts or villi. This finding could reflect an innate feeding habit, since chickens naturally consume insects when reared in free-range systems [54].

Independently of TM meal utilization, the intestinal crypts of the birds of the current trial showed greater acidic sialylated mucins staining intensity. This represents an interesting finding, since sialic acid groups have protective properties [55] and increase in acidic sialylated mucins production has been hypothesized to represent a defense strategy against mucus degradation by bacterial colonization [4]. On the contrary, similar staining intensity of the three types of mucins was observed in the intestinal villi. The variations in the proportion of the goblet cells containing the mucin types observed in the intestinal crypts instead of villi could be related to the higher presence of mucin-producing goblet cells in the first compartment, as reported by Uni et al. [56].

The intestinal crypts of both chickens fed with control and TM diets of the present research showed lower mucin staining intensity in the cecum compared with the other gut segments. This finding is in agreement with what observed by Tsirtsikos et al. [9], even if the authors provided no explanation for that result. The avian cecum has been found to be a site for fermentation and further digestion of feed (especially for breakdown of cellulose), for utilization and absorption of water and nitrogenous components, for microbial action of both beneficial and disease-causing organism, and as a site for production of immunoglobulins and antibodies [57]. As previously reported mucins enhance the propulsion of chyme, modulate nutrient absorption and protect the intestinal mucosa from enteric pathogens [6]. Since the small intestine represents the major site of nutrient absorption [58] and the ileum has been suggested to be more predisposed to bacterial colonization [4], it is possible to speculate that the mucin synthesis for nutrient metabolism and gut protection could be reduced in the cecum. Furthermore, because the cecum is blind-ended, its contents can be retained for long periods, with no need for mucin secretion to facilitate the propulsion of chyme. Therefore, the decreased mucin staining intensity in the cecal crypts observed in the present study could be considered a physiological feature related to the different anatomy and physiology of the cecum.

Independently of dietary TM meal inclusion, the intestinal villi of the free-range chickens of the present study showed greater mucin staining intensity in the ileum compared with the other gut segments. This is consistent with previous findings in chickens $[4,59]$, which demonstrated a distal increase in the density of goblet cells along the duodenal-ileal axis [59]. Forder et al. [4] suggested that the distal ileum could be a preferred region for bacterial colonization, thus explaining the need for greater protection and subsequent higher mucin synthesis.

The intestinal crypts of both birds fed with control and TM diets of the current trial showed greater mucin staining intensity in the base compared with the other crypt fragments. This is in agreement with previous studies, in which the decreased stain in the crypt tip has been suggested to be related to the process of proliferation and maturation of goblet cells $[9,56]$. Similarly, the intestinal villi showed higher mucin staining intensity in the base compared with the other villus fragments. This is in contrast with what observed by Tsirtsikos et al. [9, 10], which found higher staining intensity at the villus tip and explained this increased accumulation as a confirm for the key role of mucin in the protection of gut epithelium against luminal threats $[9,10]$. However, the process of cell proliferation in chicken intestinal epithelium has also been reported to occur along the entire length of the villus, with proliferation activity decreasing from crypt to the upper half of the villus [56]. Therefore, the higher mucin staining intensity observed at the villus base in the birds of the present study can be related to the physiological goblet cells proliferation process occurring in villus compartment. 


\section{Conclusions}

In conclusion, dietary TM meal inclusion may positively modulate the gut microbiota of the free-range chickens without influencing the intestinal morphology and mucin composition. The identification of physiological cecal community, gut morphological development and mucin dynamics also suggests that insect meal utilization does not negatively affect the gut health of the birds. Furthermore, since the rapid growth of chickens directly depends on morphological and functional integrity of the digestive tract (as herein confirmed), the gut health assessment by a post mortem multidisciplinary approach appears to be fundamental.

\section{Methods}

\section{Birds and experimental design}

The present study is a part of ongoing research work that aims to investigate the effects of TM meal inclusion on growth performance, haematochemical profile, carcass traits, histological features and gut health of free-range chickens [21]. In order to avoid unnecessary repetition of already published data, a brief summary of the experimental trial is reported below. The experimental protocol was approved by the Ethical Committee of the Department of Veterinary Sciences of the University of Turin [Italy].

A total of 140 43-days-old female Label Hubbard hybrid chickens (female: JA $57 \times$ male: S77CN, mean weight: $716.3 \pm 23.2 \mathrm{~g}$, purchased from "Aglietto Natura SRL" farm [Bianzè, VC - Italy]), a medium-growing genotype, were randomly allotted to two dietary treatments. Each of them consisted of five replicate floor pens, with 14 chicks per pen. The experimental unit was the pen. Two diets were formulated: a control corn-soybean-gluten meal-based diet $(C)$, normally used by the breeder, and an experimental diet (TM7.5), in which full-fat TM larvae meal (Gaobeidian Shannong Biology Co. Ltd., Gaobeidian, Hebei province - China) was included at $75 \mathrm{~g} / \mathrm{kg}$ in complete substitution of corn gluten meal. Details of the diets are shown in Additional file 3. Nutrients digestibility, apparent metabolisable energy and nitrogen-corrected apparent metabolisable energy were previously assessed [17]. All the birds were free-range reared under the same environmental conditions throughout the experimental trial. Feed (isonitrogenous and isoenergetic diets) and water were provided ad libitum for the whole experimental periods. The chickens (showing overall good health conditions at the beginning of the experiment) were regularly vaccinated and showed no signs of illness or mortality throughout the trial. Growth (initial and final live weight, average feed intake and feed conversion ratio) and slaughtering performance (carcass, breasts, thighs, deboned thighs, spleen, bursa of Fabricius, liver, gizzard and abdominal fat weights) were also unaffected by dietary treatments. Welfare-related assessments (footpad dermatitis score evaluation) were also performed after the experimental trial. The experiment lasted 54 days.

\section{Intestinal sampling and processing}

A total of ten chickens per treatment (two birds per pen) were randomly selected and slaughtered in a commercial abattoir at 97 days of age. Animals were euthanized by electrical stunning and bleeding. The remaining birds were slaughtered using the same euthanasia procedures and carcasses were submitted to proper disposal methods. Cecal content was collected into sterile plastic tubes that were promptly refrigerated (for a maximum of $2 \mathrm{~h}$ ) and frozen at $-80^{\circ} \mathrm{C}$ until DNA extraction. Intestinal segment samples (approximately $5 \mathrm{~cm}$ in length) of duodenum, jejunum, ileum and cecum were excised and flushed with $0.9 \%$ saline to remove all the content. The collected segments of intestine were the loop of the duodenum (duodenum), the tract before Meckel's diverticulum (jejunum), the tract before the ileocolic junction (ileum) and the apex of the caeca (cecum). Gut segments were fixed in Carnoy's and 10\% buffered formalin solutions for morphometric analysis and histochemical staining, respectively. Tissues were routinely embedded in paraffin wax blocks, sectioned at $5 \mu \mathrm{m}$ thickness and mounted on glass slides.

\section{DNA extraction and sequencing}

A pool of the cecal content from two chickens per pen (five pools per feeding group) was submitted to DNA extraction and sequencing. DNA was extracted with a commercial kit (DNAzol ${ }^{\circledR}$ Reagent, Thermo Fisher Scientific) according to the manufacturer's instructions. One $\mu \mathrm{l}$ of RNase (Illumina Inc., San Diego, CA) was added to digest RNA in the DNA samples, with an incubation of $1 \mathrm{~h}$ at $37^{\circ} \mathrm{C}$. DNA was quantified using the NanoDrop and standardized at $5 \mathrm{ng} / \mu \mathrm{l}$. Microbial diversity was studied by sequencing the amplified V3-V4 region of the $16 \mathrm{~S}$ rRNA gene by using primers and PCR conditions previously reported [60]. Samples multiplexing, library purification pooling and sequencing was carried out as described in the "16S Metagenomic Sequencing Library Preparation" guide by Illumina. Libraries were sequenced by BMR genomics (Padova, Italy) on a MiSeq platform (Illumina Italy s.r.l., Milan, Italy), leading to $250 \mathrm{bp}$, paired-end reads. After the first purification step following the Illumina sample preparation procedure, the library was combined with the sequencing adapters and dual indices using the Nextera XT Index Kit (Illumina, San Diego, USA), obtaining the multiplexed paired-end libraries. Individual libraries concentration in $\mathrm{nM}$ were calculated based on the size of amplicons by using a Bioanalyzer (Agilent) and diluted to $4 \mathrm{nM}$, denaturated with $0.2 \mathrm{~N} \mathrm{NaOH}$ and spiked with $20 \%(v / v)$ of PhiX. 


\section{Histomorphological investigations}

Carnoy-fixed and paraffin-embedded intestinal sections of ten chickens per feeding group (two birds per pen) were submitted to Haematoxylin \& Eosin staining and one slide per each intestinal segment was examined by light microscopy. Each slide was captured with a Nikon DS-Fi1 digital camera coupled to a Zeiss Axiophot microscope using a $2.5 \times$ objective lens. NIS-Elements $\mathrm{F}$ software was used for image capturing. Morphometric analysis was performed by Image ${ }^{\bullet}$-Pro Plus software. The evaluated morphometric indices were villus height (Vh, from the tip of the villus to the crypt), crypt depth (Cd, from the base of the villus to the submucosa) and the villus height to crypt depth ratio $(\mathrm{Vh} / \mathrm{Cd})$ [7]. Morphometric measurements were performed on 10 well-oriented and intact villi and 10 crypts chosen from duodenum, jejunum and ileum [8].

\section{Histochemical staining}

Formalin-fixed and paraffin-embedded intestinal sections of ten chickens per feeding group (two birds per pen) were submitted to three different histochemical staining, in order to characterize the three types of mucins [4].

Neutral mucins were identified by periodic-acid Schiff staining. Sections were brought to water, immersed in $0.5 \%$ periodic acid solution for $20 \mathrm{~min}$, washed in running tap water for $5 \mathrm{~min}$ and immersed in Schiff's reagent for a further $30 \mathrm{~min}$. Sections were successively rinsed in running tap water for $10 \mathrm{~min}$, dehydrated and mounted. Neutral mucins stained magenta [61].

Acidic sialylated mucins were identified by Alcian Blue pH 2.5 staining. Sections were brought to water, immersed in $8 \mathrm{G} \mathrm{X}$ alcian blue in $3 \%$ acetic acid solution for $30 \mathrm{~min}$, washed in running tap water for further 5 min and successively dehydrated and mounted. Acidic sialylated mucins stained blue [62].

Acidic sulfated mucins were identified by high iron diamine staining. Sections were brought to water, oxidized in $1 \%$ periodic acid solution for $10 \mathrm{~min}$ and washed in running tap water for further $5 \mathrm{~min}$. Sections were successively immersed in the high iron diamine solution $(120 \mathrm{mg}$ metadiamine, $20 \mathrm{mg}$ paradiamine and $1.4 \mathrm{ml} 10 \%$ ferric chloride in $50 \mathrm{ml}$ distilled water) for $18 \mathrm{~h}$, rinsed very rapidly in tap water, dehydrated and mounted. Acidic sulfated mucins stained purple-black [63].

\section{Mucin staining intensity}

One slide per histochemical staining for each intestinal segment was examined by light microscopy. A total of 10 crypts and 10 villi for each slide were evaluated. Crypts and villi were divided into three fragments (base, midsection and tip), according to Tsirtsikos et al. $[9,10]$. Mucin staining intensity of goblet cells was scored semiquantitatively for each fragment as follows: grade 0 for absent staining, grade 1 for mild staining, grade 2 for moderate staining and grade 3 for marked staining. The score was formulated according to that proposed by Tsirtsikos et al. $[9,10]$ for the mucus layer and depended on the number of positive goblet cells and the intensity of the staining. All the slides were assessed blinded by three observers (IB, EB and MTC) and the discordant cases were reviewed at a multi-head microscope until a consensus was reached.

\section{Bioinformatics and statistical analysis}

The experimental unit was the pen for 16S rRNA sequences and bird for morphometric and histochemical data.

Paired-end reads were first merged using FLASH software [64] with default parameters. Joint reads were further quality filtered (at Phred > Q20) using QIIME 1.9.0 software [65] and the pipeline recently described [66]. Briefly, operational taxonomic units (OTUs) were picked at $97 \%$ of similarity by means of UCLUST clustering methods [67] and representative sequences of each cluster were used to assign taxonomy using the Greengenes $16 \mathrm{~S}$ rRNA gene database (version 2013). Alpha diversity indices were calculated using the diversity function of the vegan package [68]. A pairwise t-test was used to assess differences between the theses. The diversity indices were further analyzed by pairwise comparisons using Wilcoxon rank sum test to assess differences between the dietary treatments. Weighted UniFrac distance matrices were used to perform Adonis and ANOSIM statistical tests in R environment. A filtered OTU table was generated at $0.1 \%$ abundance in at least 2 samples through QIIME. The table was then used to produce the Principal Component Analysis (PCA) in R environment (https://www.r-project.org). OTU table showed the higher taxonomy resolution reached by the $16 \mathrm{~S}$ data. When the taxonomy assignment was not able to reach the genus level, the phylum, class, order or family were displayed. Pairwise Kruskal-Wallis tests were used to find significant differences in microbial taxa abundance according to the dietary treatment. The experimental unit was the pen. $P$-values were adjusted for multiple testing and a false discovery rate $(\mathrm{FDR})<0.05$ considered as significant.

The statistical analysis of morphometric and histochemical data was performed using IBM SPSS Statistics V20.0.0 software. The influence of diet on intestinal morphometric measurements was tested using Student's $t$ test for independent samples. Morphometric data were also analyzed by means of One-way ANOVA (post hoc test: Duncan's multiple range test) to evaluate the influence of intestinal segment within each dietary treatment. Results were expressed as mean and pooled standard error of the mean (SEM). $P$ values $<0.05$ were 
considered statistically significant [21]. Histochemical data were analyzed by fitting a generalized linear model (GLM) similar to those proposed by Tsirtsikos et al. [9, 10]. The GLM allowed the mean mucin staining intensity scores to depend on linear predictors such as diet, mucin type, intestinal segment and fragment within crypts or villi through a negative binomial response probability distribution with a nonlinear link function $(\log )$. The bird within treatment effect was also included in the GLM as the repeated factor. A hybrid method for parameter estimation was used and a type III analysis with Wald chi-square test was applied to assess the model effects. Results were expressed as least squares means and SEM and the interactions between factor levels were evaluated by pairwise comparisons. $\mathrm{P}$ values $<0.05$ were considered statistically significant. Statistical analysis was performed by procedure "Generalized Linear Models".

\section{Additional files}

Additional file 1: Good's coverage and a-diversity measures of cecal microbiota of free-range chickens fed with control (C) and $7.5 \%$ inclusion level of Tenebrio molitor meal (TM7.5) diets. Description column indicates the 5 replicate pens of control (C_1, C_2 C_ C 3, C_ 4 and C_5) and 7.5\% inclusion level of Tenebrio molitor meal (TM7.5_1, TM7.5_2, TM7.5_3, TM7.5_4 and TM7.5_5) dietary treatments. (XLSX 9 kb)

Additional file 2: Relative abundance of the main bacterial phyla and genera of cecal microbiota of free-range chickens fed with control (C) and 7.5\% inclusion level of Tenebrio molitor meal (TM7.5) diets. (XLSX $10 \mathrm{~kb}$ )

Additional file 3: Ingredients and chemical composition of the experimental diets. The mineral-vitamin premix (Trevit Volatili 3.5 - Trei Rio Saliceto (RE) Italy) given values are supplied per kg: $650.000 \mathrm{IU}$ of vitamin $A ; 65.000$ IU of vitamin D3; 650 IU of vitamin $E ; 80 \mathrm{mg}$ of vitamin $\mathrm{K} ; 80 \mathrm{mg}$ of vitamin $\mathrm{B} 1 ; 150 \mathrm{mg}$ of vitamin $\mathrm{B} 2 ; 770 \mathrm{mg}$ of vitamin $\mathrm{B} 3 ; 80$ $\mathrm{mg}$ of vitamin B6; $0.5 \mathrm{mg}$ of vitamin B12; $240 \mathrm{mg}$ of pantothenic acid; $4700 \mathrm{mg}$ of betaine; $1750 \mathrm{mg}$ of Iron (II) carbonate; $1835 \mathrm{mg}$ of Magnesium oxide; $1612 \mathrm{mg}$ of Zinc oxide; $178 \mathrm{mg}$ of Copper (II) oxide; $18.3 \mathrm{mg}$ of Potassium iodide; $6.6 \mathrm{mg}$ of Sodium selenite; $4100 \mathrm{mg}$ of DL-methionine; $5500 \mathrm{mg}$ of L-lysine; $120 \mathrm{~g}$ Calcium carbonate; $450 \mathrm{~g}$ Calcium phosphate; $11.5 \mathrm{~g}$ of Sodium chloride. SFA: saturated fatty acids; MUFA: monounsaturated fatty acids; PUFA: polyunsaturated fatty acids. Other fatty acids (all less than $0.40 \mathrm{~g} / \mathrm{kg}$ DM): C12:0, C14:0, C14:1 cis9, C16:1 cis9, C18:1 cis11, C20:0, C18:3 n6, C20:1 cis9, C20:1 cis11. $\mathrm{TM}$, Tenebrio molitor; AME, apparent metabolizable energy; DM, dry matter; CP, crude protein; $\mathrm{C}=$ control; $\mathrm{TM} 7.5=7.5 \%$ inclusion level of Tenebrio molitor. (XLSX $10 \mathrm{~kb})$

\section{Abbreviations}

C: Control; Cd: Crypt depth; FDR: False discovery rate; GLM: Generalized linear model; OTUs: Operational taxonomic units; PD: Phylogenetic Diversity; TM: Tenebrio molitor; TM7.5: 7.5\% inclusion level of Tenebrio molitor; Vh: Villus height; Vh/Cd: Villus height to crypt depth ratio

\section{Acknowledgments}

The authors are grateful to Dr. Oreste Massimino, Mr. Roberto Borgogno and Mr. Aldo Pozzo - "O.R.A. Agricola Srl" (Cherasco, CN - Italy) and Mr. Alessandro Varesio and Mrs. Roberta Lacopo - "Fattoria La Fornace", Montechiaro d'Asti (At - Italy) - for their technical support. The authors are also grateful to Dr. Paolo Montersino, Mr. Dario Sola and Mr. Mario Colombano for bird care and technical support.

\section{Funding}

The research was supported by the University of Turin (2014-2015) and by the European Knowledge and Innovation Community (KIC), within the EIT Food program 'INNOPOULTRY. The poultry food chain: tackling old problems with innovative approaches (Project ID 18023). The funding bodies were involved in the animals purchase, collection of data and article processing charges.

\section{Availability of data and materials}

All data generated or analysed during this study are included in this published article [and its supplementary information files]. All the sequencing data were deposited at the Sequence Read Archive of the National Center for Biotechnology Information (SRP125741).

\section{Authors' contributions}

IB conceived the study, collected the samples, performed the histomorphological and histochemical investigations, analyzed the data, wrote the manuscript and prepared the tables and figures. IF analyzed the data and prepared the tables and figures. EB collected the samples and performed the histomorphological and histochemical investigations. EG collected the samples and performed the DNA extraction. SD and FG collected the samples. AS2 processed and cut the gut samples. LG, AS1 and LC conceived the study. MTC conceived the study and performed the histomorphological and histochemical investigations. All authors read, edited and approved the final manuscript.

\section{Ethics approval}

The experimental protocol was designed according to the guidelines of the current European and Italian laws on the care and use of experimental animals (European Directive 86,609/EEC, put into law in Italy with D.L. 116/92). Furthermore, the experimental protocol was approved by the Ethical Committee of the Department of Veterinary Sciences of the University of Turin (Italy).

\section{Consent for publication}

Not applicable.

\section{Competing interests}

The authors declare that they have no competing interests.

\section{Publisher's Note}

Springer Nature remains neutral with regard to jurisdictional claims in published maps and institutional affiliations.

\section{Author details}

${ }^{1}$ Department of Veterinary Sciences, University of Turin, Largo Paolo Braccini 2, 10095 Grugliasco, TO, Italy. ${ }^{2}$ Department of Agricultural, Forest and Food Sciences, University of Turin, Largo Paolo Braccini 2, 10095 Grugliasco, TO, Italy. ${ }^{3}$ Institute of Science of Food Production, National Research Council, Largo Paolo Braccini 2, 10095 Grugliasco, TO, Italy. ${ }^{4}$ Institute of Multidisciplinary Research on Sustainability, University of Turin, Via Accademia Albertina 13, 10100 Turin, Italy.

Received: 25 May 2018 Accepted: 9 November 2018 Published online: 04 December 2018

\section{References}

1. Kogut $M H$, Arsenault RJ. Editorial: gut health: the new paradigm in food animal production. Front Vet Sci. 2016;3:71.

2. Bailey RA. Gut health in poultry - the world within. Huntsville: Aviagen; 2013.

3. Pan D, Yu Z. Intestinal microbiome of poultry and its interaction with host and diet. Gut Microbes. 2014;5:108-19.

4. Forder RE, Howarth GS, Tivey DR, Hughes RJ. Bacterial modulation of small intestinal goblet cells and mucin composition during early posthatch development of poultry. Poult Sci. 2007;86:2396-403.

5. Wang JX, Peng KM. Developmental morphology of the small intestine of African ostrich chicks. Poult Sci. 2008:87:2629-35.

6. Forstner G, Forstner JF. Gastrointestinal mucus. In: Johnson LR, editor. Physiology of the gastrointestinal tract. New York: Raven Press; 1994. p. 1255-84.

7. Laudadio V, Passantino L, Perillo A, Lopresti G, Passantino A, Khan RU, Tufarelli $V$. Productive performance and histological features of intestinal mucosa of broiler chickens fed different dietary protein levels. Poult Sci. 2012;91:265-70. 
8. Qaisrani SN, Moquet PC, van Krimpen MM, Kwakkel RP, Verstegen MW Hendriks WH. Protein source and dietary structure influence growth performance, gut morphology, and hindgut fermentation characteristics in broilers. Poult Sci. 2014;93:3053-64.

9. Tsirtsikos P, Fegeros K, Kominakis A, Balaskas C, Mountzouris KC. Modulation of intestinal mucin composition and mucosal morphology by dietary phytogenic inclusion level in broilers. Animal. 2012a;6:1049-57.

10. Tsirtsikos P, Fegeros K, Balaskas C, Kominakis A, Mountzouris KC. Dietary probiotic inclusion level modulates intestinal mucin composition and mucosal morphology in broilers. Poult Sci. 2012b;91:1860-8.

11. Reed S, Neuman H, Glahn RP, Koren O, Tako E. Characterizing the gut (Gallus gallus) microbiota following the consumption of an iron biofortified Rwandan cream seeded carioca (Phaseolus Vulgaris L.) bean-based diet. PLoS One. 2017;12:e0182431

12. Yegani $M$, Korver DR. Factors affecting intestinal health in poultry. Poult Sci. 2008;87:2052-63.

13. FAO (Food and Agriculture Organization of the United Nations). Edible insects - future prospects for food and feed security, FAO Forestry Paper, vol. IX; 2013. p. 171.

14. van Huis A. Potential of insects as food and feed in assuring food security. Annu Rev Entomol. 2013;58:563-83.

15. Makkar HPS, Tran G, Heuzé $V$, Ankers P. State of the art on use of insects as animal feed. Anim Feed Sci Technol. 2014;197:1-33.

16. Bovera F, Piccolo G, Gasco L, Marono S, Loponte R, Vassalotti G, Mastellone V, Lombardi P, Attia YA, Nizza A. Yellow mealworm larvae (Tenebrio molitor, L.) as a possible alternative to soybean meal in broiler diets. Br Poult Sci. 2015:56:569-75.

17. De Marco M, Martínez S, Hernandez F, Madrid J, Gai F, Rotolo L, Belforti M, Bergero D, Katz H, Dabbou S, Kovitvadhi A, Zoccarato I, Gasco L, Schiavone A. Nutritional value of two insect meals (Tenebrio molitor and Hermetia illucens) for broiler chickens: apparent nutrient digestibility, apparent ileal amino acid digestibility and apparent metabolizable energy. Anim Feed Sci Technol. 2015;209:211-8.

18. Bovera F, Loponte R, Marono S, Piccolo G, Parisi G, laconisi V, Gasco L, Nizza A. Use of Tenebrio molitor larvae meal as protein source in broiler diet: effect on growth performance, nutrient digestibility and carcass and meat traits. J Anim Sci. 2016;94:639-47.

19. Biasato I, Gasco L, De Marco M, Renna M, Rotolo L, Dabbou S, Capucchio MT, Biasibetti E, Tarantola M, Bianchi C, Cavallarin L, Gai F, Pozzo L, Dezzutto D, Bergagna S, Schiavone A. Effects of yellow mealworm larvae (Tenebrio molitor) inclusion in diets for female broiler chickens: implications for animal health and gut histology. Anim Feed Sci Technol. 2017;234:253-63.

20. Biasato I, Gasco L, De Marco M, Renna M, Rotolo L, Dabbou S, Capucchio MT, Biasibetti E, Tarantola M, Sterpone L, Cavallarin L, Gai F, Pozzo L, Bergagna S, Dezzutto D, Zoccarato I, Schiavone A. Yellow mealworm larvae (Tenebrio molitor) inclusion in diets for male broiler chickens: effects on growth performance, gut morphology, and histological findings. Poult Sci. 2018:97:540-8.

21. Biasato I, De Marco M, Rotolo L, Renna M, Dabbou S, Capucchio MT, Biasibetti E, Tarantola M, Costa P, Gai F, Pozzo L, Dezzutto D, Bergagna S, Martinez S, Gasco L, Schiavone A. Effects of dietary Tenebrio molitor meal inclusion in free-range chickens. J Anim Physiol Anim Nutr. 2016; 100:1104-12.

22. Xu Y, Yang H, Zhang L, Su Y, Shi D, Xiao H, Tian Y. High-throughput sequencing technology to reveal the composition and function of cecal microbiota in Dagu chicken. BMC Microbiol. 2016;16:259.

23. Mancabelli L, Ferrario C, Milani C, Mangifesta M, Turroni F, Duranti S, Lugli GA, Viappiani A, Ossiprandi MC, van Sinderen D, Ventura M. Insights into the biodiversity of the gut microbiota of broiler chickens. Environ Microbiol. 2016;18:4727-38.

24. Ferrario C, Alessandri G, Mancabelli L, Gering E, Mangifesta M, Milani C, Lugli GA, Viappiani A, Duranti S, Turroni F, Ossiprandi MC, Hiyashi R, Mackie R, van Sinderen D, Ventura M. Untangling the cecal microbiota of feral chickens by culturomic and metagenomic analyses. Environ Microbiol. 2017;19:4771-83.

25. Oakley BB, Lillehoj HS, Kogut MH, Kim WK, Maurer JJ, Pedroso A, Lee MD, Collett SR, Johnson TJ, Cox NA. The chicken gastrointestinal microbiome. FEMS Microbiol Lett. 2014;360:100-12.

26. Wise MG, Siragusa GR. Quantitative analysis of the intestinal bacterial community in one- to three-week-old commercially reared broiler chickens fed conventional or antibiotic-free vegetable-based diets. J Appl Microbiol. 2007;102:1138-49.
27. Tillman GE, Haas GJ, Wise MG, Oakley B, Smith MA, Siragusa GR. Chicken intestine microbiota following the administration of lupulone, a hop-based antimicrobial. FEMS Microbiol Ecol. 2011;77:395-403.

28. Wei S, Morrison M, Yu Z. Bacterial census of poultry intestinal microbiome. Poult Sci. 2013;92:671-83.

29. Gong J, Si W, Forster RJ, Huang R, Yu H, Yin Y, Yang C, Han Y. 16 S rRNA gene-based analysis of mucosa-associated bacterial community and phylogeny in the chicken gastrointestinal tracts: from crops to ceca. FEMS Microbiol Ecol. 2007:59:147-57.

30. Danzeisen JL, Kim HB, Isaacson RE, Tu ZJ, Johnson TJ. Modulations of the chicken cecal microbiome and metagenome in response to anticoccidial and growth promoter treatment. PLOS One. 2011;6:e27949.

31. Stanley D, Denman SE, Hughes RJ, Geier MS, Crowley TM, Chen H, Haring VR, Moore RJ. Intestinal microbiota associated with differential feed conversion efficiency in chickens. Appl Microbiol Biotechnol. 2012; 96:1361-9.

32. Costa MC, Bessegatto JA, Alfieri AA, Weese JS, Filho JA, Oba A. Different antibiotic growth promoters induce specific changes in the cecal microbiota membership of broiler chicken. PLoS One. 2017;12:e0171642.

33. Callaway TR, Dowd SE, Wolcott RD, Sun Y, McReynolds JL, Edrington TS, Byrd JA, Anderson RC, Krueger N, Nisbet DJ. Evaluation of the bacterial diversity in cecal contents of laying hens fed various molting diets by using bacterial tag-encoded FLX amplicon pyrosequencing. Poult Sci. 2009;88: 298-302.

34. Xue Z, Zhang W, Wang L, Hou R, Zhang M, Fei L, Zhang X, Huang $H$, Bridgewater LC, Jiang Y, Jiang C, Zhao L, Pang X, Zhang Z. The bambooeating giant panda harbors a carnivore-like gut microbiota, with excessive seasonal variations. MBio. 2015;6:e00022-15.

35. Kuhn I, Katouli M, Lund A, Wallgren P, Mollby R. Phenotype diversity and stability of intestinal coliform flora in piglets during the first three months of age. Microbial Ecol Health Dis. 1993;6:101-7.

36. Stanley D, Geier MS, Hughes RJ, Denman SE, Moore RJ. Highly variable microbiota development in the chicken gastrointestinal tract. PLoS One. 2013:8:e84290.

37. Sergeant MJ, Constantinidou C, Cogan TA, Bedford MR, Penn CW, Pallen MJ. Extensive microbial and functional diversity within the chicken cecal microbiome. PLoS One. 2014;9:e91941.

38. Li Y, Xu Q, Huang Z, Lv L, Liu X, Yin C, Yan H, Yuan J. Effect of Bacillus subtilis CGMCC 1.1086 on the growth performance and intestinal microbiota of broilers. J Appl Microbiol. 2016;120:195-204.

39. Singh KM, Shah T, Deshpande S, Jakhesara SJ, Koringa PG, Rank DN, Joshi CG. High through put 165 rRNA gene-based pyrosequencing analysis of the fecal microbiota of high FCR and low FCR broiler growers. Mol Biol Rep. 2012;39:10595-602.

40. Pryde SE, Duncan SH, Hold GL, Stewart CS, Flint HJ. The microbiology of butyrate formation in the human colon. FEMS Microbiol Lett. 2002; 217:133-9.

41. Yang L, Liu S, Ding J, Dai R, He C, Xu K, Honaker CF, Zhang Y, Siegel P, Meng $\mathrm{H}$. Gut microbiota co-microevolution with selection for host humoral immunity. Front Microbiol. 2017:8:1243.

42. Onrust L, Ducatelle R, Van Driessche K, De Maesschalck C, Vermeulen K, Haesebrouck F, Eeckhaut V, Van Immerseel F. Steering endogenous butyrate production in the intestinal tract of broilers as a tool to improve gut health. Front Vet Sci. 2015;2:75.

43. Liu C, Finegold SM, Song Y, Lawson PA. Reclassification of Clostridium coccoides, Ruminococcus hansenii, Ruminococcus hydrogenotrophicus, Ruminococcus luti, Ruminococcus productus and Ruminococcus schinkii as Blautia coccoides gen. Nov., comb. nov., Blautia hansenii comb. nov., Blautia hydrogenotrophica comb. nov., Blautia luti comb. nov., Blautia producta comb. nov., Blautia schinkii comb. nov. and description of Blautia wexlerae sp. nov., isolated from human faeces. Int J Syst Evol Microbiol. 2008;58: 1896-902.

44. Sunkara LT, Jiang W, Zhang G. Modulation of antimicrobial host defense peptide gene expression by free fatty acids. PLoS One. 2012;7:e49558.

45. Gillespie MJ Stanley D, Chen H, Donald JA, Nicholas KR, Moore RJ, Crowley TM. Functional similarities between pigeon 'milk' and mammalian milk: induction of immune gene expression and modification of the microbiota. PLoS One. 2012;7:e48363.

46. Chang CL, Chung CY, Kuo CH, Kuo TF, Yang CW, Yang WC. Beneficial effect of Bidens pilosa on body weight gain, food conversion ratio, gut Bacteria and coccidiosis in chickens. PLoS One. 2016;11:e0146141. 
47. Wrigley DM. Inhibition of Clostridium perfringens sporulation by Bacteroides fragilis and short-chain fatty acids. Anaerobe. 2004;10:295-300.

48. Swatson HK, Gous R, lij PA, Zarrinkalam R. Effect of dietary protein level, amino acid balance, and feeding level on growth, gastrointestinal tract, and mucosal structure of the small intestine in broiler chickens. Anim Res. 2002; 51:501-15.

49. Oliveira MC, Rodrigues EA, Marques RH, Gravena RA, Guandolini GC, Moraes VMB. Performance and morphology of intestinal mucosa of broilers fed mannan-oligosaccharides and enzymes. Arq Bras Med Vet Zootec. 2008;60: $442-8$.

50. Miles RD, Butcher GD, Henry PR, Littell RC. Effect of antibiotic growth promoters on broiler performance, intestinal growth parameters, and quantitative morphology. Poult Sci. 2006;85:476-85.

51. Ferraris RP, Villenas SA, Diamond J. Regulation of brush-border enzyme activities and enterocyte migration rates in mouse small intestine. Am J Phys. 1992;262:G1047-59.

52. de Verdal H, Mignon-Grasteau S, Jeulin C, Le Bihan-Duval E, Leconte M, Mallet S, Martin C, Narcy A. Digestive tract measurements and histological adaptation in broiler lines divergently selected for digestive efficiency. Poult Sci. 2010;89:1955-61.

53. Iji PA, Saki A, Tivey DR. Body and intestinal growth of broiler chicks on a commercial starter diet. 1. Intestinal weight and mucosal development. Br Poult Sci. 2001;42:505-13.

54. Zuidh of MJ, Molnar CL, Morley FM, Wray TL, Robinson FE, Khan BA, AlAni L, Goonewardene LA. Nutritive value of house fly (Musca domestica) larvae as a feed supplement for Turkey poults. Anim Feed Sci Technol. 2003;105:225-30.

55. Corfield AP, Wagner SA, Clamp JR, Kriaris MS, Hoskins LC. Mucin degradation in the human colon: production of sialidase, sialate Oacetylesterase, $\mathrm{N}$-acetylneuraminate lyase, arylesterase, and glycosulfatase activities by strains of fecal bacteria. Infect Immun. 1992;60:3971-8.

56. Uni Z, Platin R, Sklan D. Cell proliferation in chicken intestinal epithelium occurs both in the crypts and along the villus. J Comp Physiol B. 1998;168: 241-7.

57. Clench MH, Mathias JR. The avian cecum - a review. Wilson Bull. 1995;107: 93-121.

58. Dingle JG. Module 3: nutrient procurement and processing, study book: poultry husbandry 1. Toowoomba: DEC, UCSQ; 1991.

59. Uni Z, Smirnov A, Sklan D. Pre- and posthatch development of goblet cells in the broiler small intestine: effect of delayed access to feed. Poult Sci. 2003;82:320-7.

60. Klindworth A, Pruesse E, Schweer T, Peplies J, Quast C, Horn M, Glöckner FO. Evaluation of general $16 S$ ribosomal RNA gene PCR primers for classical and next-generation sequencing-based diversity studies. Nucleic Acids Res. 2013;41:e1.

61. McManus JFA. Histological demonstration of mucin after periodic acid. Nature. 1946;158:202.

62. Pearse AGE. Histochemistry: Theoretical and Applied. 2nd ed. Churchill, Livingstone: London; 1960.

63. Spicer SS. Diamine methods for differentiating mucosubstances histochemically. J Histochem Cytochem. 1965;13:211-34.

64. Magoč T, Salzberg SL. FLASH: fast length adjustment of short reads to improve genome assemblies. Bioinformatics. 2011;27:2957-63.

65. Caporaso JG, Kuczynski J, Stombaugh J, Bittinger K, Bushman FD, Costello EK, Fierer N, Peña AG, Goodrich JK, Gordon Jl, Huttley GA, Kelley ST, Knights $D$, Koenig JE, Ley RE, Lozupone CA, McDonald D, Muegge BD, Pirrung M, Reeder J, Sevinsky JR, Turnbaugh PJ, Walters WA, Widmann J, Yatsunenko T, Zaneveld J, Knight R. QIIME allows analysis of high-throughput community sequencing data. Nat Methods. 2010;7:335-6.

66. Ferrocino I, Bellio A, Romano A, Macori G, Rantsiou K, Decastelli L, Cocolin L. RNA-based amplicon sequencing reveals microbiota development during ripening of artisanal versus industrial lard d'Arnad. Appl Environ Microbiol. 2017;83. https://doi.org/10.1128/AEM.00983-17.

67. Edgar RC. Search and clustering orders of magnitude faster than BLAST. Bioinformatics. 2010;26:2460-1.

68. Dixon P. VEGAN, a package of $\mathrm{R}$ functions for community ecology. J Veg Sci. 2003;14:927-30.

\section{Ready to submit your research? Choose BMC and benefit from:}

- fast, convenient online submission

- thorough peer review by experienced researchers in your field

- rapid publication on acceptance

- support for research data, including large and complex data types

- gold Open Access which fosters wider collaboration and increased citations

- maximum visibility for your research: over $100 \mathrm{M}$ website views per year

At BMC, research is always in progress.

Learn more biomedcentral.com/submissions 